Swarthmore College

Works

11-16-2009

\title{
Observation Of A Helical Self-Organized State In A Compact Toroidal Plasma
}

C. D. Cothran

Michael R. Brown

Swarthmore College,doc@swarthmore.edu

Timothy G. Gray , '01

M. J. Schaffer

G. Marklin

Follow this and additional works at: https://works.swarthmore.edu/fac-physics

Part of the Physics Commons

Let us know how access to these works benefits you

\section{Recommended Citation}

C. D. Cothran; Michael R. Brown; Timothy G. Gray , '01; M. J. Schaffer; and G. Marklin. (2009). "Observation Of A Helical Self-Organized State In A Compact Toroidal Plasma". Physical Review Letters. Volume 103, Issue 21. DOI: 10.1103/PhysRevLett.103.215002

https://works.swarthmore.edu/fac-physics/101

This work is brought to you for free by Swarthmore College Libraries' Works. It has been accepted for inclusion in Physics \& Astronomy Faculty Works by an authorized administrator of Works. For more information, please contact myworks@swarthmore.edu. 


\title{
Observation of a Helical Self-Organized State in a Compact Toroidal Plasma
}

\author{
C. D. Cothran, ${ }^{1, *}$ M. R. Brown, ${ }^{1}$ T. Gray, ${ }^{1}$ M. J. Schaffer, ${ }^{2}$ and G. Marklin ${ }^{3}$ \\ ${ }^{1}$ Swarthmore College, Swarthmore, Pennsylvania 19081, USA \\ ${ }^{2}$ General Atomics, San Diego, California 92186, USA \\ ${ }^{3}$ University of Washington, Seattle, Washington 98195, USA \\ (Received 18 September 2009; published 16 November 2009)
}

\begin{abstract}
A nonaxisymmetric stable magnetohydrodynamic (MHD) equilibrium within a prolate cylindrical conducting boundary has been produced experimentally. It has $m=1$ azimuthal symmetry, helical distortion, and flat $\lambda$ profile, all in agreement with the computed magnetically relaxed minimum magnetic energy Taylor state. Despite varied initial conditions determined by two helicity injectors on the device, this same equilibrium consistently emerges as the final state. These results therefore describe a new example of self-organization in an MHD plasma.
\end{abstract}

DOI: 10.1103/PhysRevLett.103.215002

PACS numbers: 52.30.Cv, 52.55.Ip

Some magnetohydrodynamic (MHD) plasmas at low $\beta$ (i.e., those with magnetic forces much larger than kinetic pressure gradients) exhibit the remarkable characteristic that they settle to a particular stable equilibrium state determined not by the initial conditions, but only by the shape of the boundary (presumed to be a perfectly conducting rigid wall) and some global characteristics such as total flux or current [1,2]. These plasmas are said to exhibit magnetic relaxation, a behavior which makes them a member of a broader class of self-organizing physical systems [3] which spontaneously develop large scale order, including, for example, two-dimensional fluids which form large, coherent vortex structures out of an initially turbulent state disordered at all spatial scales [4].

Taylor first recognized that in order for the magnetically relaxed state to be independent of the initial preparation of the plasma, the dynamics must be constrained to conserve the total magnetic helicity $K=\int \vec{A} \cdot \vec{B} d V$, where the integral is taken over the total plasma volume. The inclusion of some resistivity in the plasma is essential. The dynamics of ideal MHD (no resistivity) conserve the helicity of any flux tube within the plasma; with resistivity, the helicity (twist and linkage) of flux tubes are altered by the relaxation dynamics, thus destroying the details of the initial state. This happens much faster than the global resistive time scale at which the total helicity $K$ changes. Therefore, unlike the infinite number of ideal MHD invariants associated with the individual flux tube helicities, only the total helicity may be regarded a good dynamical invariant. The relaxation dynamics quickly dissipate excess magnetic energy (assuming a $\beta=0$ plasma the only free energy is magnetic), bringing the plasma to the preferred state characterized by minimum magnetic energy $W$ consistent with the same total helicity $K$ as was initially present within the conducting boundary.

Formally, the minimization of magnetic energy with the helicity conservation constraint requires that the magnetic structure of a relaxed state satisfy:

$$
\vec{\nabla} \times \vec{B}=\lambda \vec{B}
$$

or, equivalently, that $\mu_{0} \vec{J}=\lambda \vec{B}$, where $\lambda$ is a constant determined by the boundary conditions. This is a $\beta=0$ force-free MHD equilibrium $\vec{J} \times \vec{B}=0$ which demands that $\vec{J}$ is everywhere parallel to $\vec{B}$. The critical distinction for relaxed states is the spatial invariance of $\lambda$; this property directly reveals the insensitivity of the plasma to the initial conditions. Without relaxation, the final equilibrium obtained under ideal MHD would still be force free but not unique as the memory of the initial state would be preserved in the spatial dependence of $|\vec{J}| /|\vec{B}|$.

Relaxation theory describes qualitatively and in some aspects quantitatively both reversed field pinch (RFP) plasmas [5], which have a torus shaped conducting boundary, and spheromak plasmas [6-9], which are a member of the class of compact toroidal [10] (CT) plasmas having a topologically spherical boundary (typically realized experimentally as a closed cylinder). An early, important validation of Taylor's theory was the explanation of the spontaneous reversal of the toroidal field in the RFP and accurate calculation of its threshold. Aside from expected deviations near the wall, it is well established that the magnetic structures of axisymmetric equilibria for both the RFP and spheromak agree well with the predictions of Taylor relaxation theory. Helically distorted nonaxisymmetric relaxed states, however, are predicted for both types of plasmas, for values of the pinch parameter $\Theta>1.57$ in the RFP [2] and for aspect ratios $L / R>1.67$ in the CT $[11,12]$, but have not previously been identified experimentally.

This Letter (1) describes a stable, low $\beta$ nonaxisymmetric $\mathrm{CT}$ equilibrium produced experimentally within a prolate cylindrical conducting boundary with aspect ratio $L / R=3.0$; (2) compares the results to the computed Taylor state; and (3) identifies this CT as the magnetically relaxed, or self-organized, state. Internal magnetic probe measurements show its toroidal (azimuthal) symmetry is 
dominantly an $m=1$ mode and its axial structure is helically deformed, in agreement with the Taylor state structure. The measured $\lambda$ profile is spatially uniform, as predicted for magnetically relaxed plasmas, and is consistent with the computed value of $\lambda$ for the Taylor state. Two independent helicity injectors permit the initial state of the plasma to contain one or two spheromaks. Despite these very different initial conditions, a CT with the characteristics described above consistently emerges in the final state. These results therefore provide the first identification of a self-organized nonaxisymmetric MHD plasma.

Nonaxisymmetric CTs were produced at the Swarthmore Spheromak Experiment (SSX) [13]. Figure 1 shows a sketch of the experiment. Pulsed power applied to coaxial magnetized plasma guns (helicity injectors) produce fully ionized hydrogen plasma spheromaks at either end of the device. A copper cylindrical shell $(3 \mathrm{~mm}$ thick wall) defines the flux conserving boundary of the plasma at radius $R=20 \mathrm{~cm}$ and length $L=63 \mathrm{~cm}$, giving an aspect ratio of approximately 3.0 (this shell is split at the midplane for diagnostic access, but the two halves are electrically connected). The internal magnetic probes and quadrature interferometry diagnostic for electron density have been described previously [14]; in addition a fast high spectral resolution ion Doppler spectroscopy (IDS) instrument [15] monitors carbon (impurity) $T_{i}$ and fluid velocity at the midplane. The electron temperature was not measured for these experiments, but vacuum ultraviolet spectroscopy of similar SSX plasmas has recently been used to determine $T_{e} \approx 15 \mathrm{eV}$ [16]. All of these measurements are made with
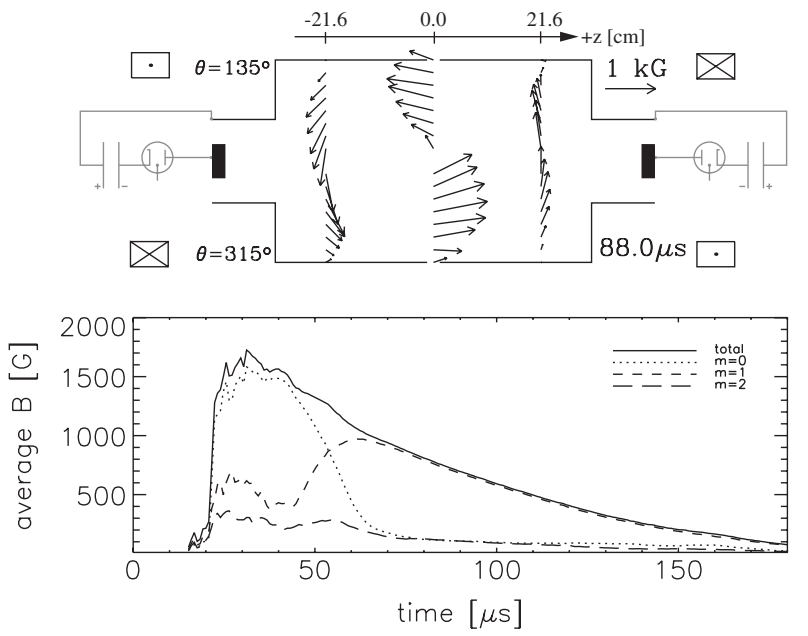

FIG. 1. (top) Sketch of the SSX device showing the helicity injectors (capacitor banks switched across coaxial magnetized electrodes), magnetic probe locations with sample data (an identical set of six probes define another $r-z$ plane orthogonal to the one shown above), and the $L / R=3.0$ cylindrical conducting boundary. (bottom) Time dependence of the average (RMS over all $96 \vec{B}$ measurements) magnetic field strength of the azimuthal Fourier modes showing the dominance of the $m=1$ component for $t \gtrsim 70 \mu \mathrm{s}$. time resolution and acquisition rate that is faster than the MHD time scale of the plasma.

The internal magnetic structure is measured at three planes orthogonal to the geometric axis $\hat{z}: z=0.0 \mathrm{~cm}$ (midplane) and $z= \pm 21.6 \mathrm{~cm}$. In each plane, four linear probes are inserted radially with equal toroidal spacing; at eight radial locations spaced at $2.5 \mathrm{~cm}$ intervals along the length of each probe, the induced EMF in three orthogonal coils is integrated to measure $\vec{B}$. The placement permits the toroidal Fourier decomposition of the magnetic structure into the two lowest toroidal mode numbers $m=0$ and $m=$ 1 , plus a partially resolved $m=2$.

Figure 1(b) compares the average strength of these mode amplitudes as two right-handed spheromaks of nearly the same helicity merge and evolve into the nonaxisymmetric state. Pulsed power is applied at $t \equiv 0 \mu \mathrm{s}$, and the two expanding spheromak plasmas cross the probes at $z=$ $\pm 21.6 \mathrm{~cm}$ by $t \approx 25 \mu \mathrm{s}$. The axisymmetric $m=0$ mode dominates for $t \lesssim 50 \mu \mathrm{s}$, when the plasma grossly resem-
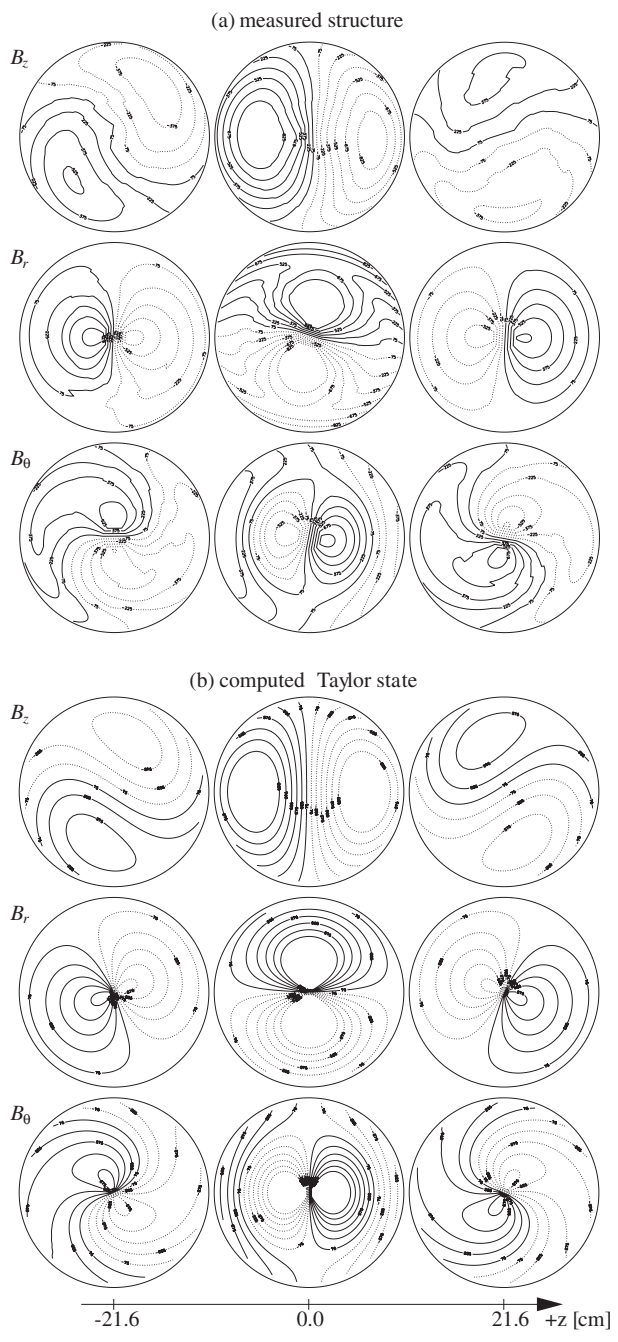

FIG. 2. Contour plots of $B_{z}, B_{r}$, and $B_{\theta}$ determined (a) experimentally for $t=88 \mu \mathrm{s}$ and (b) computed for the Taylor relaxed state. Isosurfaces are separated by 150 G. Solid (dotted) lines are positive (negative) values. 
bles two driven spheromaks. Transition from an axisymmetric to nonaxisymmetric structure is evident during the interval from $t \approx 40 \mu \mathrm{s}$ to $t \approx 70 \mu \mathrm{s}$. The time dependence of the magnetics data as well as the IDS data show the plasma to be very dynamic prior to and especially during the transition (usually initiated by tilting motion). After the transition, the $m=1$ mode is clearly dominant and there is remarkably little MHD activity; the nonaxisymmetric magnetic structure is stable and simply decays in amplitude with a time constant of about $45 \mu \mathrm{s}(e-$ folding time of $B$ ). Typical parameters of this quiescent equilibrium state are $B \approx 1.0 \mathrm{kG}, n_{e} \approx 4 \times 10^{14} / \mathrm{cm}^{3}$, $T_{i} \approx 8 \mathrm{eV}, T_{e} \approx 15 \mathrm{eV}$. The plasma is therefore low $\beta \approx$ 0.1 , fully magnetized $\left(\rho_{i} \approx 0.7 \mathrm{~cm} \ll R, L\right)$, and well described by MHD.

Figure 2(a) presents the detailed magnetic structure of the nonaxisymmetric equilibrium in each of the three circular cross sections defined by the set of probes at $z=$ $0.0, \pm 21.6 \mathrm{~cm}$ measured at $t=88 \mu \mathrm{s}$. The azimuthal Fourier decomposition obtained from the probe data is used to obtain $\vec{B}$ for all $r, \theta$ needed for the contours shown in Fig. 2. To do this, the partially resolved $m=2$ is ignored; this is justified by the small strength of the $m=$ 2 mode shown in Fig. 1(b).

The Taylor state structure for $L / R=3.0$ computed using the analysis described in Refs. [11,12] is shown in Fig. 2(b). Briefly, the representation $\vec{B}=\hat{z} \times \vec{\nabla} \psi+$ $1 / \lambda \vec{\nabla} \times(\hat{z} \times \vec{\nabla} \psi)$ makes Eq. (1) a Helmholtz equation $\nabla^{2} \psi+\lambda^{2} \psi=0$. The value of $\lambda$ and the complex coefficients $a_{n}$ in the separation of variables expansion

$$
\psi=\sum_{n=1} a_{n} J_{m}\left(k^{\prime} r\right) \sin k z e^{i m \theta}+a_{0} r^{m} e^{i \lambda z} e^{i m \theta},
$$

where $k=n \pi / L$ and $k^{\prime}=\left(\lambda^{2}-k^{2}\right)^{1 / 2}$, are determined by applying the boundary condition $B_{r}(r=R)=0$. This is effectively an eigenmode problem. In general, there is a discrete spectrum of eigenvalues $\lambda$ which are determined only by the shape of the boundary for CT plasmas. Since Taylor's constrained minimization procedure requires $W=(\lambda / 2) K$ for CT plasmas, the eigenmode with the smallest $\lambda$ corresponds to the minimum energy relaxed state. For aspect ratios $L / R \lesssim 1.67$, the smallest eigenvalue corresponds to an axisymmetric $(m=0)$ eigenmode; these are the Bessel function (spheromak) solutions. For $L / R \gtrsim 1.67$ the eigenmode with $m=1$ toroidal symmetry has the smallest eigenvalue. The relaxed state for a prolate $\mathrm{CT}$ is therefore predicted to be nonaxisymmetric with pure $m=1$ toroidal symmetry.

Detailed agreement of the experiment and the calculated Taylor state structure is quite good. The only free parameter in the computation is the total helicity, which was adjusted to give the best visual agreement with the experimental data. An additional global azimuthal rotation is necessary in principle, but this angle is evidently small; the CT tends to align itself along a pair of magnetic probes at the midplane. The dominant $m=1$ mode in the measured structure bears out the prediction of a pure $m=1$ azimuthal symmetry of the Taylor state. The positive (solid) and negative (dotted) contours for all components clearly show sign inversion for any two points $(r, \theta)$ and $(r$, $\theta+\pi)$, as required for an $e^{i \theta}$ dependence.

A helical distortion is plainly evident in the structure of this CT. Previous work [17] did not recognize the helical deformation and described the structure to be that of a spheromak tilted on its side. Moving along $z$ successively through each plane of data, the contours in Fig. 2 rotate by about $\pi$, giving a normalized axial wave number $k R \approx 1.6$ which is consistent with the $k$ spectrum in Eq. (2). The positive and negative lobes of $B_{z}$ in Fig. 2 can be interpreted as opposite sides of a single closed loop of flux that twists on itself, or, more simply, a tilted and twisted spheromak. This structure is effectively the Taylor "double helix" solution for the cylindrical RFP approximation with no axial flux, which has two opposing, infinite length flux bundles twisted with a pure $k R \approx 1.23$ pitch (i.e., $e^{i k z+i \theta}$ dependence), with finite length effects.

The remarkably good agreement between the measured and computed equilibrium is a strong indicator that this plasma is magnetically relaxed. This may be tested further by examining the $\lambda$ profile which is predicted to be spatially uniform for a relaxed plasmas. Presuming force-free conditions $(\beta \approx 0)$, the local value of $\lambda$ may be computed using the $z$ component of $\mu_{0} \vec{J}=\lambda \vec{B}$ expressed in cylindrical coordinates so that

$$
\lambda=\frac{1}{B_{z}}\left[\frac{1}{r} \frac{\partial}{\partial r}\left(r B_{\theta}\right)+\frac{1}{r} \frac{\partial B_{r}}{\partial \theta}\right] .
$$
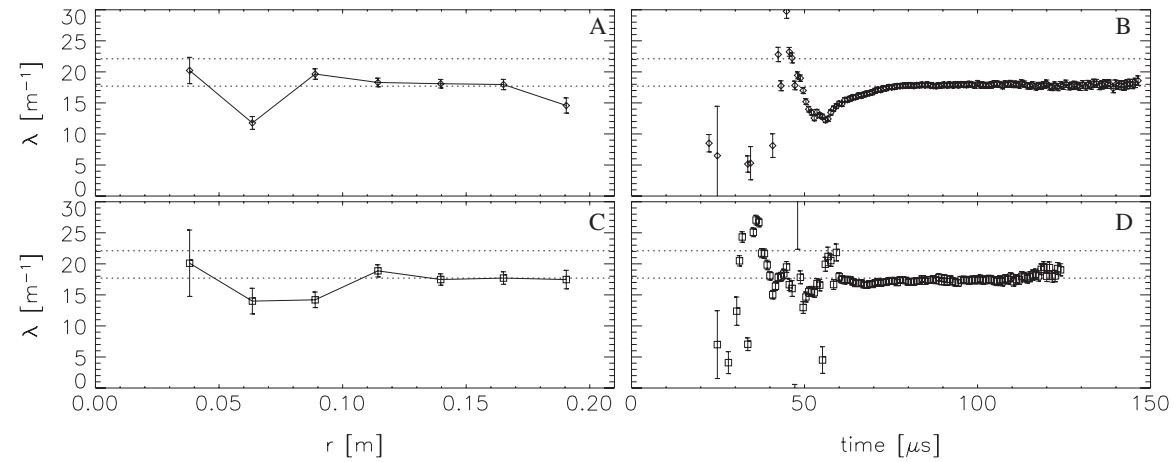

215002-3
FIG. 3. Measurements of $\lambda$ following relaxation from the two-spheromak initial state (a),(b) and from the single spheromak initial state (c),(d). The radial profiles of $\lambda$ (a),(c) are consistent with the Taylor state value (dotted line at $\lambda=$ $\left.17.7 \mathrm{~m}^{-1}\right)$. The radial average of $\lambda(\mathrm{ex}-$ cluding the two values at smallest $r$ ) stays very near the Taylor state value throughout the decay of the nonaxisymmetric self-organized state (b),(d). 
The $m=1$ mode $\theta$ dependence in the data can be exploited to evaluate the $\theta$ derivative.

Figure 3(a) shows the $\lambda$ profile determined by applying Eq. (3) to the measured midplane $(z=0)$ magnetic structure of Fig. 2(a). The profile is reasonably flat, as anticipated by relaxation theory. Furthermore, the experimental value of $\lambda$ is found to be in excellent agreement with the predicted value $\lambda=17.7 \mathrm{~m}^{-1}$ (lower dotted line). For comparison, the $m=0$ spheromak Bessel function eigenmode for $L / R=3.0$ has $\lambda=19.9 \mathrm{~m}^{-1}$; it is therefore not the minimum energy state for this aspect ratio, nor is it compatible with the $m=1$ structure of the observed relaxed state. The next $m=1$ eigenmode has $\lambda=22.0 \mathrm{~m}^{-1}$, which is clearly inconsistent with the observed $\lambda$ profile (upper dotted line).

The time dependence of the radially averaged value of $\lambda$ is shown in Fig. 3(b). Only after the plasma begins to settle to the helical relaxed state with its pure $m=1$ toroidal symmetry $(t \gtrsim 70 \mu \mathrm{s})$ does Eq. (3) give a good determination of $\lambda$. The value of $\lambda$ is then extremely steady, although Fig. 1(b) shows the plasma decays substantially by $t \approx 150 \mu \mathrm{s}$. The helicity and magnetic energy must therefore be decaying at the same rate.

Note that the confinement of this CT may be unexpectedly good despite its nonaxisymmetric structure. The lifetime of this CT from Fig. 1(b) is as good or better than the lifetime of axisymmetric plasmas produced at SSX; in addition, there is no indication of relaxation oscillations in Figs. 3(b) and 3(d).

Finally, when only one of the pulsed power banks is discharged, the initial plasma contains a single spheromak which also quickly succumbs to the tilting instability and evolves to the same helically distorted relaxed state described above. Figures 3(c) and 3(d) show the results for the $\lambda$ profile and time dependence to be substantially identical to those for Figs. 3(a) and 3(b). All of the preceding analysis applied to single spheromak experiments yields equally good comparisons to the computed Taylor state as for the two-spheromak initial state experiments. The detailed magnetic structure measurements are omitted for brevity.

In general, instabilities drive a self-organizing physical system to a preferred (i.e., self-organized) state which is independent of the initial system configuration except for global parameters. The helically distorted, nonaxisymmetric stable CT plasma described above may be regarded as a self-organized, or magnetically relaxed, state since it emerges as the dynamical end point of tilt instabilities for either the one- or two-spheromak initial plasmas. The excellent agreement with the predicted Taylor state suggests the total helicity is the important global parameter constraining the self-organization processes, although there is no evidence of the magnetic turbulence postulated by Taylor's theory for rapid access to the relaxed state. Furthermore, self-organized states generally are structured at the longest scale lengths of the system. The twospheromak initial configuration clearly has a scale length determined by half the system size, while the nonaxisymmetric $\mathrm{CT}$ has relaxed within the full length of the cylinder. To the extent that the $\lambda$ for the one- and two-spheromak initial configurations are at least those of the Bessel function spheromak values $\lambda=19.9 \mathrm{~m}^{-1}$ and $\lambda=21.8 \mathrm{~m}^{-1}$, respectively, the plasma is evolving in both cases to decrease $\lambda$ to the value $\lambda=17.7 \mathrm{~m}^{-1}$ of the nonaxisymmetric CT; i.e., toward a magnetic structure with larger characteristic spatial scale length.

To summarize, this Letter provides the first experimental description of the magnetic self-organization of a nonaxisymmetric CT plasma. For both one- and two-spheromak initial conditions, the plasma spontaneously evolves to the same helically distorted stable MHD equilibrium with $m=$ 1 symmetry. The flat $\lambda$ profile shows that this CT is magnetically relaxed. Good agreement with the Taylor state suggests robust invariance of the total helicity. The structure can be described as the Taylor "double helix" with finite length effects, or as a tilted and twisted spheromak.

This research was supported by grants from the U.S. Department of Energy and the National Science Foundation. The authors thank V.S. Lukin for carefully reading this manuscript.

*Present address: Global Strategies Group (North America), Crofton, MD 21114, USA. cothran.c@gmail.com

[1] J. B. Taylor, Phys. Rev. Lett. 33, 1139 (1974).

[2] J. B. Taylor, Rev. Mod. Phys. 58, 741 (1986).

[3] A. Hasegawa, Adv. Phys. 34, 1 (1985).

[4] D. Montgomery et al., Phys. Fluids A 4, 3 (1992).

[5] H. A. B. Bodin and A. A. Newton, Nucl. Fusion 20, 1255 (1980).

[6] M. N. Rosenbluth and M. N. Bussac, Nucl. Fusion 19, 489 (1979).

[7] T. R. Jarboe et al., Phys. Rev. Lett. 51, 39 (1983).

[8] T. R. Jarboe, Plasma Phys. Controlled Fusion 36, 945 (1994).

[9] P.M. Bellan, Spheromaks (Imperial College Press, London, 2000).

[10] H. Alfvén, in Proceedings of the Second United Nations International Conference on the Peaceful Uses of Atomic Energy (United Nations, Geneva, Switzerland, 1958), Vol. 31, p. 3.

[11] A. Bondeson et al., Phys. Fluids 24, 1682 (1981).

[12] J. M. Finn, W. Manheimer, and E. Ott, Phys. Fluids 24, 1336 (1981).

[13] M. R. Brown et al., Phys. Plasmas 6, 1717 (1999).

[14] C. D. Cothran et al., Phys. Plasmas 10, 1748 (2003).

[15] C. D. Cothran et al., Rev. Sci. Instrum. 77, 063504 (2006).

[16] V. Chaplin et al., Phys. Plasmas 16, 042505 (2009).

[17] T. R. Jarboe et al., Phys. Rev. Lett. 45, 1264 (1980). 